\title{
NON SEMINOMATOUS MIXED GERM CELL TUMOR OF TESTIS WITH A LARGE ABDOMINAL AND RETROPERITONEAL EXTENSION: A CASE REPORT
}

\author{
Kayastha R, Pradhan S, Acharya R, Aryal M, Shrestha A, Tuladhar AS
}

Department of Radiodiagnosis, Nepal Medical College Teaching hospital, Attarkhel, Gokarneshwor-8, Kathmandu, Nepal

\begin{abstract}
Primary testicular germ cell tumors (PGCT) can be classified as seminomatous and non-seminomatous germ-cell tumor (NSGCT) types. Mixed germ cell tumors (MGCT), a subtype of NSGCT, contain more than one germ cell components. Here, we present a rare case of a MGCT composed of yolk sack tumor and teratoma which had a continuous large abdominal and retroperitoneal extension. A 43 years old male presented with complaints of discomfort and swelling over the right inguinoscrotal region. Ultrasonography (USG) showed a large ill-defined heteroechoic mass in the right inguinoscrotal region with vascularity and without separate visualization of right testis. Subsequent contrast enhanced Computed Tomography (CT) showed large enhancing mass in the right scrotal sac which was continuous with large abdominopelvic and retroperitoneal mass through the right inguinal canal. Tru-Cut biopsy of the scrotal mass showed MGCT with yolk sac and teratoma component. Patient underwent 6 cycles of chemotherapy followed by Right Radical Inguinal Orchidectomy.
\end{abstract}

\section{KEYWORDS}

Non seminomatous germ cell tumor, Mixed germ cell tumor, Ultrasound, Computed Tomography

\section{CORRESPONDING AUTHOR}

Dr. Rumita Kayastha

Registrar,

Department of Radiodiagnosis, Nepal Medical College

Teaching Hospital, Attarkhel, Gokarneshwor- 8,

Kathmandu, Nepal

Email: rumikayastha@gmail.com

Orcid ID: 0000-0003-3146-4687

DOI: https://doi.org/10.3126/nmcj.v22i1-2.30047 


\section{INTRODUCTION}

Primary testicular tumors can be classified as seminomatous and non-seminomatous germcell tumor (NSGCT) types. Mixed germ cell tumors (MGCT) contain more than one germ cell components and are much more common than any of the pure histologic forms representing 32\%-60\% of all germ cell tumors. ${ }^{1}$ Non seminomatous germ cell tumors presents with heterogenous imaging features and multiple histologic patterns often seen in men at their 30s. ${ }^{1}$ Approximately $50 \%$ of germ cell tumor are seminoma and approximately $50 \%$ are NSGCT. ${ }^{2}$ Of NSGCT, $33 \%$ are mixed germ cell tumors, $10 \%$ are pure embryonal carcinoma, $4 \%$ are teratoma, $1 \%$ are yolk sac tumor and $0.3 \%$ are choriocarcinoma. ${ }^{2}$ Cryptoorchidism, family history, microlithiasis, radiation, infection and hypospadias are risk factors for testicular cancer. Patients with testicular tumor usually present with painless testicular swelling, discomfort, backpain and abdominal discomfort. Specific diagnosis of mixed type of NSGCT is difficult and challenging in imaging because of various imaging features. Here we present a rare case of a MGCT composed of yolk sac tumor and teratoma with a large continuous abdominal and retroperitoneal mass communicating through right inguinal canal.

\section{CASE REPORT}

A 43 years old male patient presented to the Department of Emergency, Nepal Medical College Teaching Hospital with history of discomfort and swelling over the right inguinoscrotal region. Ultrasound showed a large ill-defined vascular heteroechoic mass in the right inguinoscrotal region which was continuous with a large abdominal and retroperitoneal lesion.
Lab investigations showed raised tumor markers Alpha-Fetoprotein (AFP): 2100ng/ml, Beta-HCG: 6,75 IU/ml, Lactate Dehydrogenase (LDH) : 900 U/l.

Contrast enhanced CT showed a large heterogeneously enhancing mass within the right scrotal sac measuring $15 \times 12 \times 9.0 \mathrm{~cm}$. Right testis could not be visualized separately. Large heterogenously enhancing soft tissue density mass measuring $25 \times 15 \times 15 \mathrm{~cm}$ was also noted involving the abdominopelvic cavity and retroperitoneal region which was continuous with the right scrotal mass through the right inguinal canal. The mass was abutting the abdominal aorta for a length of $78 \mathrm{~mm}$ without evidence of encasement, attenuation or intra luminal filling defects. There was encasement and invasion of infrarenal portion of inferior venacava with complete attenuation of its lumen. Tuft of vessels were noted within the lesion. The mass was supplied by the branches of inferior epigastric artery. Superiorly, it was extending upto the inferior surface of pancreas; medially was abutting the anterior surface of rectum with maintained fat plane. The mass was abutting the medial surface of right psoas and iliacus muscle with doubtful fat plane. There was encasement and narrowing of bilateral mid and lower ureters without separate delineation of bilateral distal ureters leading to bilateral mild hydroureteronephrosis. There were few enhancing lymph nodes involving the aortocaval, precaval, and right para-aortic lymph nodes. With these atypical imaging features differential diagnosis of primary testicular tumor with abdominal and retroperitoneal extension with regional lymphadenopathy and lymphoma was considered.

Subsequent tru-cut biopsy of scrotal mass and abdominal mass revealed NSGCT with yolk sac
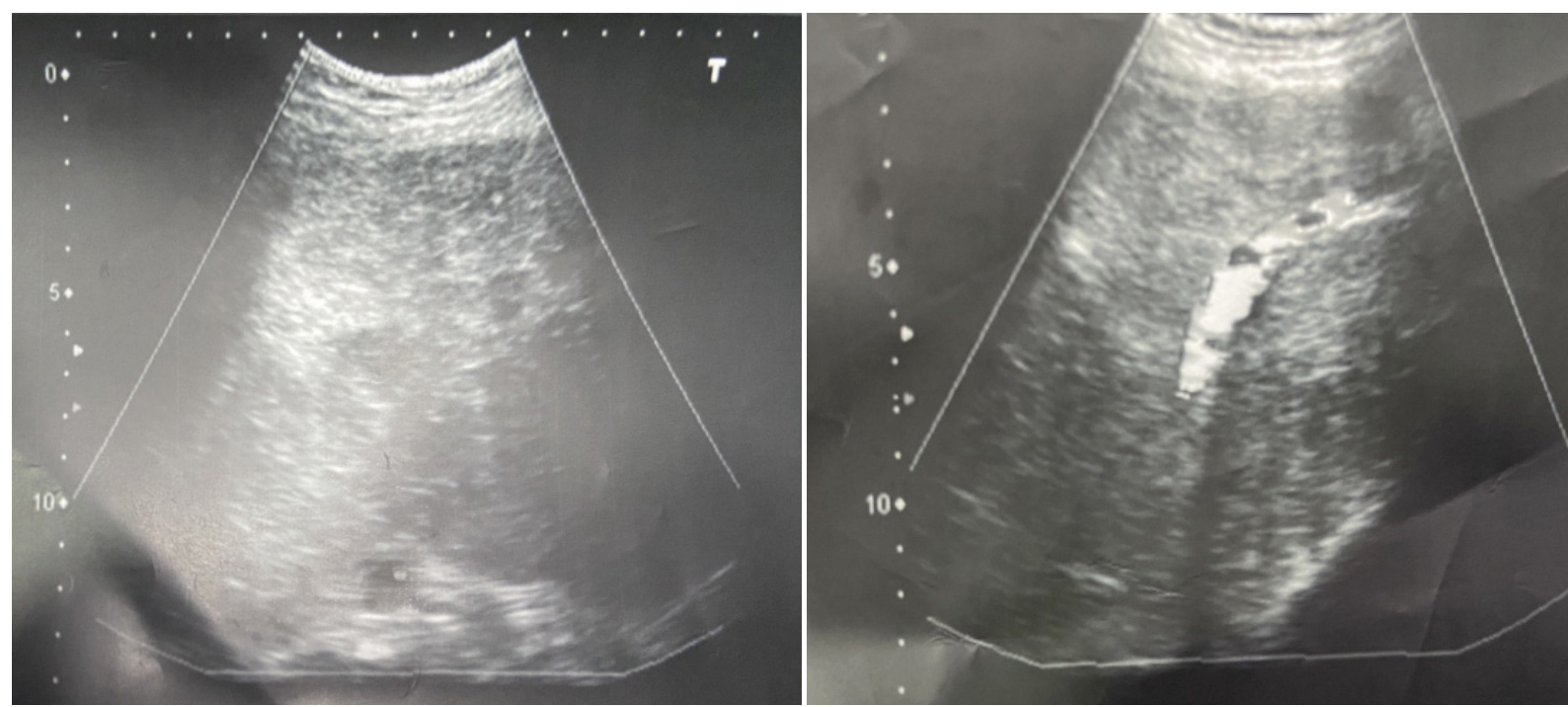

Fig.1: USG of right scrotal region showing a large heteroechoic lesion with internal vascularity without calcifications or cystic spaces. 
and teratoma component. After the completion of 6 cycles of chemotherapy follow up CECT was done which showed areas of calcification and necrosis. Then the patient underwent right Radical Inguinal Orchidectomy. Intraoperatively, a large highly vascular multilobulated mass was seen in the right testis extending up to the deep inguinal ring with invasion of tunica vaginalis at multiple sites. The mass was resected upto the deep inguinal ring.

\section{HISTOPATHOLOGY:}

Microscopic examination showed fibrocartilagenous tissue revealing derivatives of all the three germ layers. Ectodermal, endodermal and mesodermal components comprising of squamous epithelial nests, gastrointestinal mucosa (columnar epithelium interspersed with goblet cells) and cartilage was noted. Areas of dispersed tumor cells in reticular microcystic
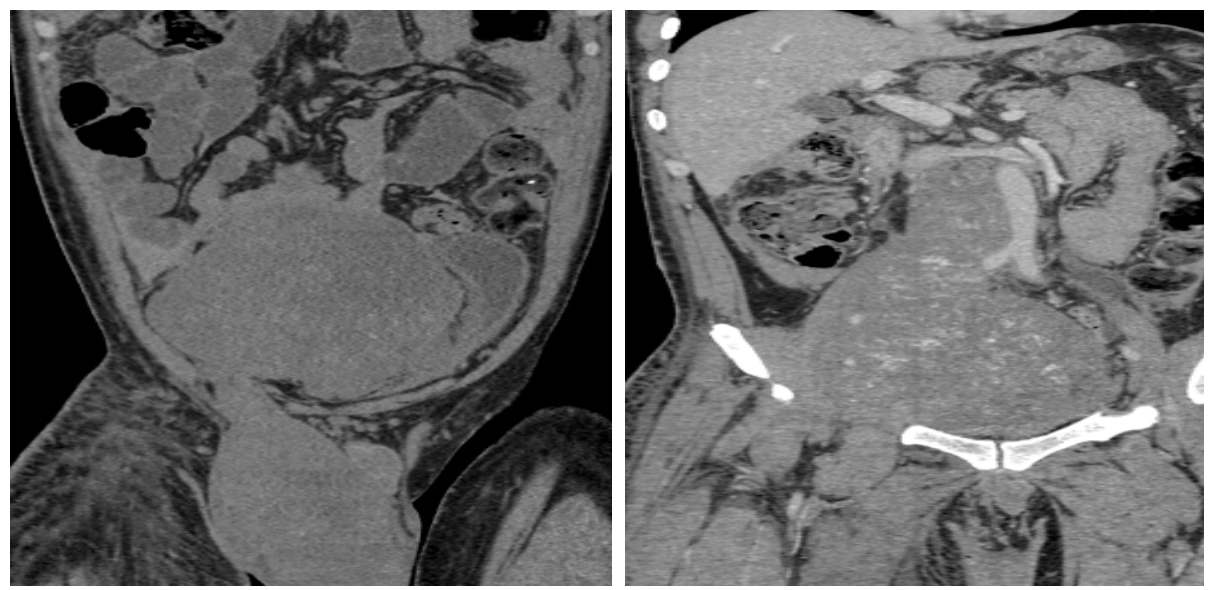

Fig. 2: Plain and Contrast enhanced CT scan (coronal section) showing heterogeneously enhancing large scrotal mass extending into the abdomino-pelvic cavity.

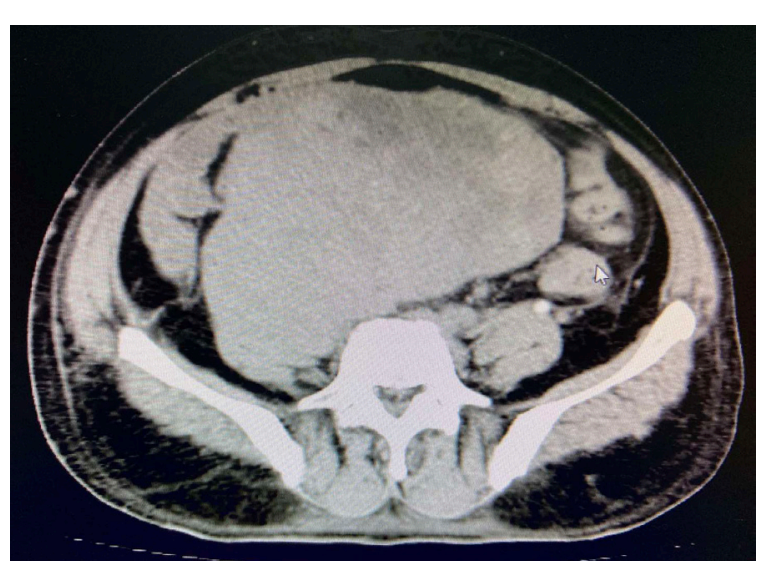

Fig. 3: Axial CECT showing large abdominal and retroperitoneal mildly enhancing soft tissue mass. pattern consisting of irregular loose spaces and anastomosing thin cord and tubules lined by flat to cuboidal cells was noted. The microcystic pattern was characterized by prominent cytoplasmic vacuoles to small cystic spaces with papillary cluster of cells projecting into the lumen. Myxoid stroma was noted in some areas. There were areas of hemorrhage, necrosis along with mild lymphocytic infiltrates, numerous foci of scattered germ cells with nuclear enlargement and cytoplasmic clearing resembling intratubular germ cells.

On immunohistochemical analysis showed diffuse positive Vimetin, focal positive SALL4, AFP, SMA, Glypican 3, Negative S100, GFAP, highlights the epithelial elements (CK AE1/AE3) and 20-25\% Ki67.
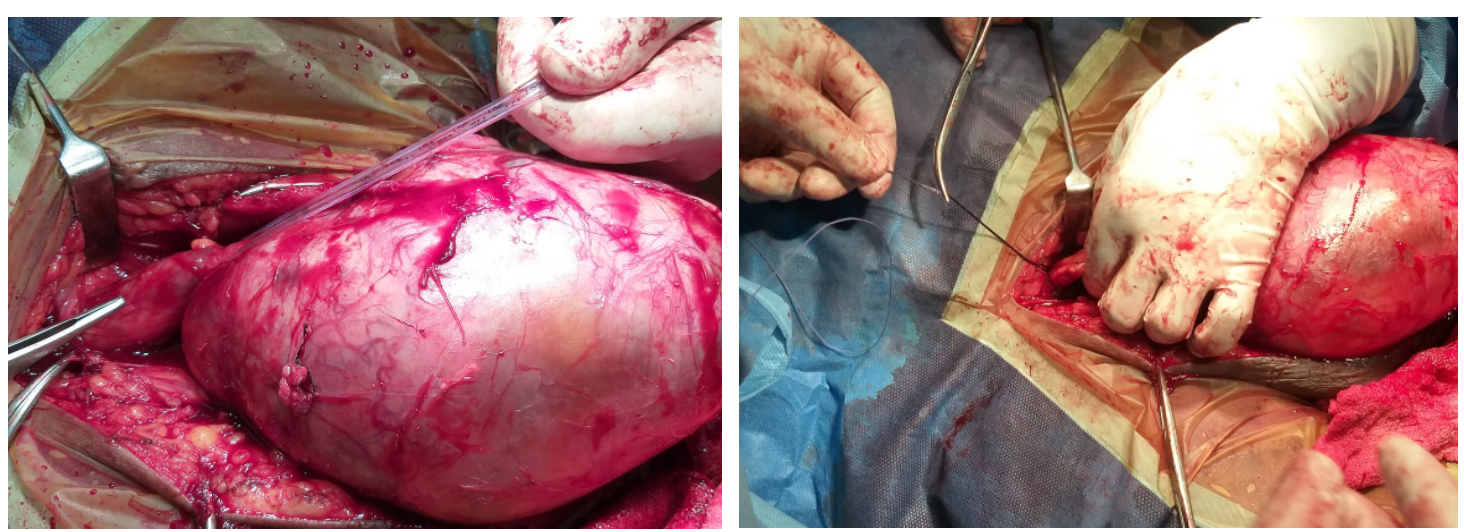

Fig. 4: Intraoperative images showing a large well defined inguinoscrotal mass upto the deep inguinal ring. 


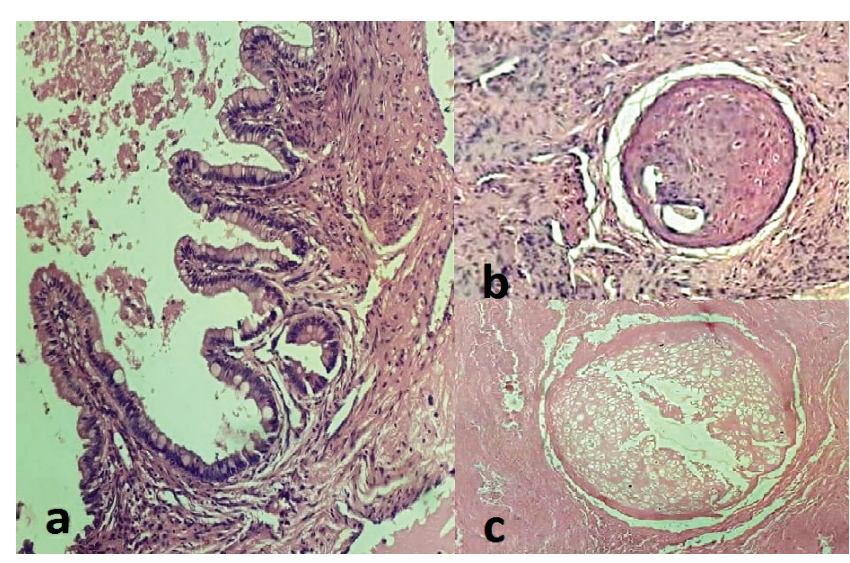

Fig 5: Mature teratoma component (H E stain 400x): (a) columnar epithelium interspersed with goblet cell. (b) Nest of squamous epithelium (c) Foci of cartilage.

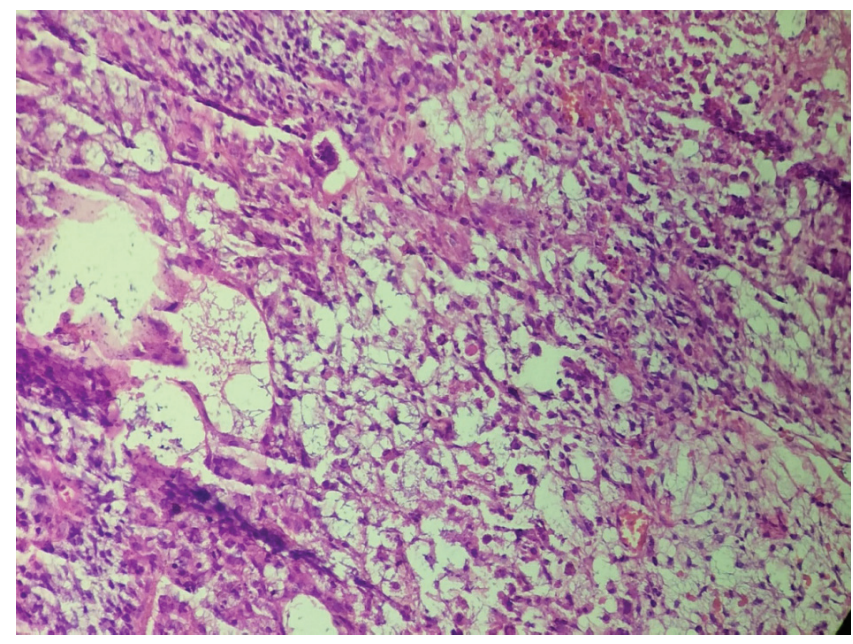

Fig 6: Yolk sac component with a reticular pattern that consists of irregular anastomosing channels, forming loose cystic spaces containing myxoid material (H E stain 400x).

\section{DISCUSSION}

Testicular tumors are relatively rare tumors. It makes up approximately $2 \%$ of all malignant cancers in men and account for up to $10 \%$ of all malignant diseases occurring within the male genitourinary system. ${ }^{1}$ In young men approximately $95 \%$ of testicular cancer are germ cell tumors and only 5\% are sex cord stromal tumors. And among germ cell tumors nearly 50 $\%$ are seminoma and $50 \%$ are non seminomatous germ cell tumors. ${ }^{1-3}$ Of the NSGCTs, 33\% are mixed germ cell tumors, $10 \%$ are pure embryonal carcinoma, $4 \%$ are teratoma, $1 \%$ are yolk sac tumors and $0.3 \%$ are choriocarcinoma. ${ }^{1,2}$ Among mixed, most common combination is teratoma and embryonal cell carcinoma. ${ }^{2}$ Patient with testicular tumor usually present with painless testicular mass/ discomfort, backpain and abdominal discomfort due to retroperitoneal mass. ${ }^{3}$ NSGCT are thought to arise from totipotent cell line that undergo varying degree of dedifferentiation into embryonal, teratoma, yolk sac or choriocarcinoma components, thus account for their heterogeneity whereas seminoma are thought to arise from unipotent cell line, so are homogenous. ${ }^{4}$ The spread of testicular tumors is both by lymphatic and haematogenous routes. ${ }^{4,5}$ Abdominal retroperitoneal lymph nodes are considered regional lymph nodes. ${ }^{3-5}$ Tumors involving the left testicle typically spread first to left paraaortic lymph nodes just below the left renal vein. ${ }^{6}$ Tumors involving the right testicle typically spread first to paracaval, precaval, and retrocaval lymph nodes. ${ }^{6,7}$

Seminomas are often hypoechoic and homogeneous. Homogeneous appearance is reflected at gross pathologic and histologic analysis, where uniform cells are seen. ${ }^{4,5}$ In comparison, NSGCTs are more commonly heterogeneous in echotexture, with most demonstrating cystic spaces. ${ }^{4,5,8}$ Calcifications are also seen much more frequently in NSGCTs than in seminomas. ${ }^{4} \mathrm{CT}$ or $\mathrm{MRI}$ is the investigation of choice which will reveal the size, site, extension, vascularity, content, invasion, relation, characterization and stage of tumor. ${ }^{9-11}$ USG, CT and MRI show heterogenous appearance in imaging features. ${ }^{4,7}$ Although NSGCTs have distinct histological entities, in general, they have similar radiographic appearances.

The imaging features of germ cell tumor in our case showed a large testicular mass with abdominal and retroperitoneal extensions which appears heterogeneous on plane $\mathrm{CT}$ and shows heterogeneous enhancement. However, there was no evidence of cystic spaces within this mass as described in different literature. So, the imaging features of NSGCT alone is challenging. Different patterns of NSGCT are difficult to distinguish through radio imaging. In our case, large abdominopelvic and retroperitoneal component and its contiguous relation with inguinoscrotal mass has increased confusion whether retroperitoneal component is metastasis or direct extension. There are many literature and case reports describing the abdominal and retroperitoneal lymphadenopathy which is common in testicular tumors. To our knowledge, there has not been any reported case of MGCTs with continuous extension into the abdominal and retroperitoneal compartments. There was no evidence of distant metastases in our case.

But seminomatous and NSGCT can be differentiated through radioimaging and is necessary to differentiate as mode of treatment is different for both.,12 Seminomatous germ cell tumors are radiosensitive whereas NSGCTs are 
not. ${ }^{12,13}$ Histological study of germ cell tumor helps to specify the type of NSGCT. ${ }^{14}$ Among the different types, NSGCT mixed type is common. ${ }^{14,15}$ However, in our case we could not describe the particular type of testicular mass as it didn't show the characteristic imaging features of NSCGTs. There was no evidence of calcifications or any necrotic areas within the mass. Though most of the literatures show that the combination of teratoma and embryonal cell tumor is common, some studies have shown association of teratoma with yolk sac, which is similar to this case study. ${ }^{14,15}$

In conclusion, testicular cancer is relatively rare and can have variable presentations.
Inguinoscrotal swelling with heterogenous radiographic features help in differentiating NSGCTs with other similar lesions. Orchidectomy with retroperitoneal dissection and chemotherapy is the treatment of choice with a variable prognosis. NSGCTs can also present with large abdominal and retroperitoneal component.

\section{ACKNOWLEDGEMENTS}

We would like to thank Dr. Rakesh Pathak for providing information on histopathologic findings of this case.

\section{REFERENCES}

1. Stamatiou K, Papadopoulos P, Perlepes G et al. Mixed germ cell tumor of the testicle with ravdomuosarcomatous component: a case report. Cases J 2009; 2: 9299.

2. Tsili AC, Tsampoulas C, Giannakopoulos X et al. MRI in the histologic characterization of testicular neoplasms. AJR Am J Roentgenol 2007; 189: 331-7

3. Lim YJ, Jeong MJ, Bae BN et al. Seminoma in undescended testis. Abdom Imaging 2008; 33: 2413.

4. Woodward PJ, Sohaey R, O’Donoghue et al. Tumors and tumor like lesions of the testis: radiologicpathologic correlation. RadioGraphics 2002; 22: 189-216.

5. Schwerk WB, Schwerk WN, Rodeck G. Testicular tumors: prospective analysis of real-time US patterns and abdominal staging. Radiology 1987; 164: 369-74.

6. Walsh TJ, Croughan MS, Schembri M et al. Increased risk of testicular germ cell cancer among infertile men. Arch Intern Med 2009; 169: 351-6.

7. Hilton S, Herr HW, Teitcher JB et al. CT detection of retroperitoneal lymph node metastases in patients with clinical stage I testicular nonseminomatous germ cell cancer: assessment of size and distribution criteria. Am J Roentgenol 1997; 169: 521-5

8. Schwerk WB, Schwerk WN, Rodeck G. Testicular tumors: prospective analysis of real-time US patterns and abdominal staging. Radiology 1987; 164: 369-74.

9. Mohrs OK, Thoms H, Egner T et al. MRI of patients with suspected scrotal or testicular lesions: diagnostic value in daily practice. Am J Roentgenol 2012; 199: 609-15.

10. Tsili AC, Argyropoulou MI, Giannakis D et al. MRI in the characterization and local staging of testicular neoplasms. Am J Roentgenol 2010; 194: 682-9.

11. Tsili AC, Argyropoulou MI, Astrakas LG et al. Dynamic contrast-enhanced subtraction MRI for characterizing intratesticular mass lesions. Am J Roentgenol 2013; 200: 578-85

12. Ulbright TM. Germ cell tumors of the gonads: a selective review emphasizing problems in differential diagnosis, newly appreciated, and controversial issues. Mod Pathol 2005; 18: 61-79

13. Krag Jacobsen G, Barlebo H, Olsen J et al. Testicular germ cell tumours in Denmark.: Acta Radiol Oncol 1984; 23: 239-47.

14. Mosharafa AA, Foster RS, Leibovich BC et al. Histology in mixed germ cell tumor. J Urol 2004; 171: 1471-3.

15. Raman JD, Nobert CF, Goldstein M. Increased incidence of testicular cancer in men presenting with infertility and abnormal semen analysis. $J$ Urol 2005; 174: 1819-22. 